\title{
DEVELOPMENT AND EVALUATION OF A SLIDING MODE OBSERVER-BASED SENSOR FAULT RECONSTRUCTION ALGORITHM FOR DC MOTORS
}

\author{
Kwangseok $\mathrm{Oh}^{1}$, Jaho $\mathrm{Seo}^{2 *}$ \\ ${ }^{1}$ Department of Mechanical Engineering, Hankyong National University, Anseong-si, Korea \\ ${ }^{2}$ Department of Automotive, Mechanical and Manufacturing Engineering, \\ University of Ontario Institute of Technology, Oshawa, Canada \\ *Jaho.Seo@uoit.ca
}

\begin{abstract}
This paper presents a sensor fault reconstruction algorithm for DC motors using a sliding mode observer. The sliding mode observer was designed through modeling the DC motor dynamics with actual system parameters and applying a linear transformation matrix for state transformation. In the designed observer, a discrete injection term was considered to achieve asymptotic stability of the error dynamics. Evaluations were conducted using an actual DC motor test platform to validate the performance of the proposed fault reconstruction algorithm. Evaluation results show that the developed algorithm can reconstruct a sensor fault signal to represent an actual fault signal with small errors.
\end{abstract}

Keywords-sliding mode observer; voltage sensor fault; injection term; DC motor; fault reconstruction

\section{INTRODUCTION}

Different types of motors such as DC, induction, and step motors have been widely used in dynamic motion control applications. Among them, the DC motor possesses attractive features for motion control in various industrial sectors that include excellent speed control, delivery of high starting torque, and easy and simple control design, etc.

A sensor is an essential component of DC motor systems since it can detect physical quantities including voltage, torque, and angular displacement whose information is used to monitor the performance of motor systems and to control them. In addition, state estimation techniques such as Kalman filtering can contribute to improving the performance of motor control systems by estimating the rotational velocity. However, since its control performance can be degraded by unexpected sensor faults, various studies have investigated the areas of fault detection, isolation, and identification in motor control applications.

Adouni [1] proposed an artificial neural network-based architecture that can enable fault detection, isolation, and identification to minimize fault alarms for a DC motor by generating robust residuals. In the study of $\mathrm{Lu}$ [2], a simple and easily implemented algorithm was developed, which can run on an embedded system for the online fault diagnosis of motor bearing. For the safety of motor systems, the detection of an online inter-turn fault is essential. This is because an inter-turn fault is the second most commonly observed but the most severe fault. To tackle this issue, Singh [3] presented infrared thermography-based online and non-invasive techniques to detect the presence of an inter-turn fault in motor drives. The sensor fault-resilient control using a high order sliding mode observer was developed for DC servomotors to detect sensor faults [4]. However, this approach only dealt with a fixed threshold and may potentially deteriorate the closed-loop performance due to highly increased control inputs for speed tracking. In the existent studies, there exist various methods for fault detection of hardware and sensors using the model-based algorithms that require relatively accurate model parameters. However, it is not easy to obtain such accurate model parameters. As an alternative solution, an artificial intelligencebased algorithm has been considered but reliable and sufficient training data sets are prerequisites to achieving high performance.

To address the above limitations, this study proposes a methodology to reconstruct signal faults of a voltage sensor in DC motors by applying a sliding mode observer. For this, a mathematical dynamic equation of the DC motor was modeled and a sliding mode observer was designed based on the dynamic equation. Transformation and distribution matrices were designed by considering the stability conditions of the observer. A performance evaluation of the developed sensor fault reconstruction algorithm was conducted using an actual DC motor test platform.

The rest of the paper is organized as follows. Section 2 describes the fault reconstruction algorithm with a sliding mode observer. Section 3 presents the results of performance evaluation. Finally, concluding remarks are provided in Section 4. 


\section{SLIDING Mode ObSERVER-BASED FAUlT RECONSTRUCTION}

\section{A. Dynamics of a DC motor system}

Figs. 1-2 show the actual DC motor test platform used in this study and its electrical circuit diagram, respectively.

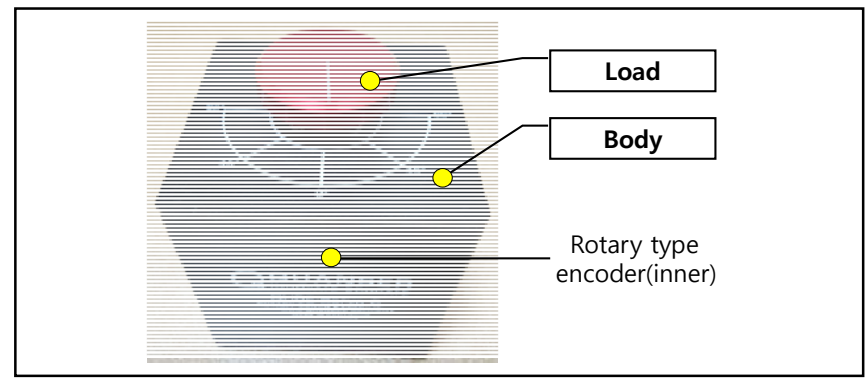

Figure 1. Actual DC motor test platform

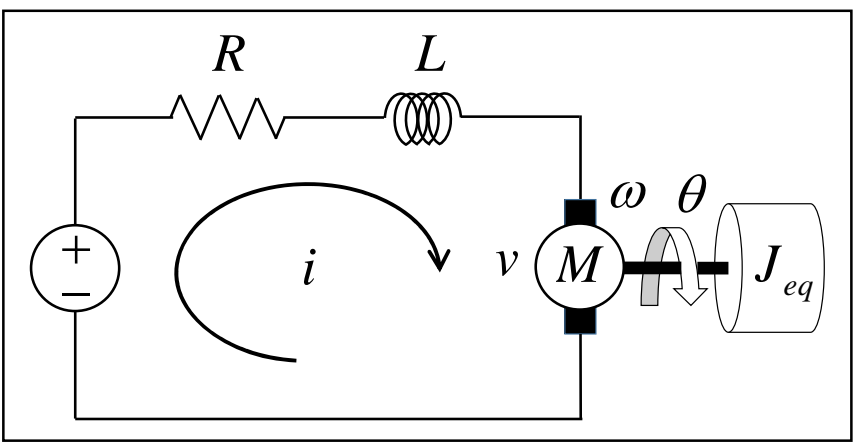

Figure 2. The electrical circuit of a DC motor system

The considered test platform was equipped with a rotary type encoder to measure the angular displacement. The platform was compatible with the Matlab/Simulink software. The dynamic equations of the DC motor in Fig. 1 can be presented by the following Eq.(1)-(3) that originate from the Kirchhoff's voltage law.

$$
\begin{gathered}
v(t)-R i(t)-L \frac{d i}{d t}-k \omega(t)=0 . \\
i(t)=\frac{v(t)-k_{b} \omega(t)}{R}, L \ll R . \\
\dot{\omega}(t)+\frac{k_{t} k_{b}}{J_{e q} R} \omega(t)=\frac{k_{t}}{J_{e q} R} v(t) .
\end{gathered}
$$

where $v, i, \omega$ represent the voltage input, current, and angular velocity, respectively. $R, L, J_{e q}, k_{b}, k_{t}$ are the resistor, inductance, equivalent inertia, backEMF(electromotive force) constant, and torque constant, respectively. In this study, the parameters provided by the manufacturer were used for an evaluation of the designed fault reconstruction algorithm. The parameters used in the study are given in Table 1.
TABLE I. PARAMETERS USED FOR MODELING OF DC MOTOR DYNAMICS

\begin{tabular}{|c|c|c|c|}
\hline Parameters & Description & Value & Unit \\
\hline$R$ & Resistance & 6.3 & $\Omega$ \\
\hline$L$ & Inductance & 0.85 & $\mathrm{mH}$ \\
\hline$J_{e q}$ & Equivalent inertia & $21.67 \times 10^{-6}$ & $\mathrm{kgm}^{2}$ \\
\hline$k_{b}$ & Back EMF & 0.036 & $\mathrm{Vs} / \mathrm{rad}$ \\
\hline$k_{t}$ & Torque constant & 0.036 & $\mathrm{Nm} / \mathrm{A}$ \\
\hline
\end{tabular}

\section{B. Sliding mode observer for fault reconstruction}

A sliding mode observer was adopted for the sensor fault reconstruction in the considered DC motor system. The following equations include internal system states (state-space) and outputs derived from the motor dynamics that were used to design a sliding mode observer.

$$
\begin{gathered}
\frac{d}{d t}\left[\begin{array}{l}
\theta(t) \\
\omega(t)
\end{array}\right]=\left[\begin{array}{cc}
0 & 1 \\
0 & -A
\end{array}\right]\left[\begin{array}{l}
\theta(t) \\
\omega(t)
\end{array}\right]+\left[\begin{array}{l}
0 \\
B
\end{array}\right] v(t)=A_{O}\left[\begin{array}{l}
\theta(t) \\
\omega(t)
\end{array}\right]+B_{O} v(t) \\
y(t)=C x(t)=\left[\begin{array}{ll}
1 & 1
\end{array}\right]\left[\begin{array}{l}
\theta(t) \\
\omega(t)
\end{array}\right]
\end{gathered}
$$

where $A$ and $B$ are $\frac{k_{t} k_{b}}{J_{e q} R}$ and $\frac{k_{t}}{J_{e q} R}$, respectively. Based on the linear transformation matrix in Eq. (6) that consists of the output matrix $C$ and its null space, the above state-space equation can be transformed with output and new state variables.

$$
T=\left[\begin{array}{c}
\operatorname{Null}(C)^{T} \\
C
\end{array}\right] .
$$

The observer dynamics is defined in Eq. (7) and (8) using an injection term and its distribution matrix.

$$
\begin{gathered}
\dot{\hat{x}}(t)=A_{\text {sys }} \hat{x}(t)+B_{\text {input }} v(t)+G_{n} v_{\text {in }}(t) . \\
\hat{y}(t)=C \hat{x}(t) .
\end{gathered}
$$

where the matrix $G_{n}$ was designed as $\left[\begin{array}{ll}L & -I\end{array}\right]^{T}$ to guarantee asymptotic stability for the state and output errors. $v_{i n}$ is the discrete injection term as an observer input. Using the equations of (4) and (7), the error dynamics can be derived with respect to the error state $e(t)=\hat{x}(t)-x(t)$ as follows.

$$
\dot{e}(t)=A_{s y s} e(t)+G_{n} v_{i n}(t) .
$$


Through a linear transformation using the matrix $C$, the derived error dynamics can be partitioned as follows.

$$
\begin{gathered}
\dot{e}_{1}(t)=A_{s y s, 1} e_{1}(t)+A_{s y s, 2} e_{y}(t)+L v_{i n}(t) . \\
\dot{e}_{y}(t)=A_{s y s, 3} e_{1}(t)+A_{s y s, 4} e_{y}(t)-v_{i n}(t) .
\end{gathered}
$$

To design a sliding mode observer, the injection term can be defined using the output error $e_{y}$ in Eq. (12).

$$
v_{i n}=-\rho \operatorname{sign}\left(e_{y}\right)
$$

It is assumed that the absolute value of $A_{s y s, 3} e_{1}(t)+A_{s y s, 4} e_{y}(t)$ in Eq. (4) as a disturbance is bounded. By following the assumption and eta-reachability condition [5], the output error can converge to zero in finite time. Eqs. (13) and (14) show inequality conditions for the bounded disturbance and magnitude of the computed injection term for stability.

$$
\begin{gathered}
\left|A_{s y s, 3} e_{1}(t)+A_{s y s, 4} e_{y}(t)\right| \leq L_{b} . \\
\rho=L_{b}+\eta+\frac{\alpha}{\sqrt{2}} .
\end{gathered}
$$

After the output error converges to zero by the injection term in Eq. (11), the output errors can be negligible in Eq. (10). Then, the equivalent injection can be computed and the error dynamics for the state in Eq. (11) is rewritten as follows.

$$
\begin{gathered}
v_{i n, e q}(t)=A_{s y s, 3} e_{1}(t) . \\
\dot{e}_{1}(t)=\left(A_{s y s, 1}+L A_{s y s, 3}\right) e_{1}(t) .
\end{gathered}
$$

If the gain $L$ in the matrix $G_{n}$ from Eq. (7) is selected to have the system matrix in Eq. (4) be negative definite, the state error can converge to zero asymptotically. Additionally, the injection term can maintain the error near zero despite the existence of disturbance such as a fault signal.

The key concept of the fault reconstruction is to reconstruct a fault signal with an equivalent injection term after having the convergence of error to zero. The following equations describe the error dynamics with an input fault signal.

$$
\begin{aligned}
& \dot{e}_{1}(t)=A_{\text {sys }, 1} e_{1}(t)+A_{s y s, 2} e_{y}(t)+L v_{\text {in }}(t)-B_{\text {input }, 1} f_{i n}(t) . \\
& \dot{e}_{y}(t)=A_{\text {sys }, 3} e_{1}(t)+A_{s y s, 4} e_{y}(t)-v_{\text {in }}(t)-B_{\text {input }, 2} f_{\text {in }}(t) .
\end{aligned}
$$

If the state $\left(e_{1}\right)$ and output errors $\left(e_{y}\right)$ converge to zero, the above equations can be rewritten as Eq. (19) and (20).

$$
\begin{aligned}
& 0=L v_{\text {in }}(t)-B_{\text {input }, 1} f_{\text {in }}(t) . \\
& 0=-v_{\text {in }}(t)-B_{\text {input }, 2} f_{\text {in }}(t) .
\end{aligned}
$$

By utilizing the above equations, the reconstructed input fault can be obtained as given in Eq. (21).

$$
f_{\text {in }}(t)=B_{\text {input }, 1}^{-1} L v_{\text {in }}(t)=-B_{\text {input }, 2}^{-1} v_{\text {in }}(t) .
$$

As a condition to reconstruct an actual fault, Eq. (22) needs to be satisfied.

$$
L=-B_{\text {input }, 1} B_{\text {input }, 2}^{-1} .
$$

Using the DC motor dynamics (Eq. (1)-(3)), linear transformation matrix (Eq. (6)), and condition for fault reconstruction (Eq. (22)), it is observed that an input fault of the DC motor system can be reconstructed without the information of parameter $A$. Therefore, the back-EMF constant does not affect the fault reconstruction performance.

The following Eq. (23) presents the system $\left(A_{O}\right)$ and input matrices $\left(B_{O}\right)$ of the DC motor dynamic equation. In Eq. (24), the gain $L$ was designed to generate the conditions for fault reconstruction and stability in Eq. (25) on which eigenvalues in Eq. (16) are always negative, and thus the state error dynamics can be asymptotically stable.

$$
\begin{gathered}
A_{\text {sys }}=T A_{o} T^{-1}=\left[\begin{array}{cc}
\frac{A-1}{2} & \frac{A-1}{2 \sqrt{2}} \\
\frac{A}{\sqrt{2}} & \frac{A}{\sqrt{2}}
\end{array}\right], B_{\text {input }}=T B_{o}=\left[\begin{array}{c}
\frac{B}{\sqrt{2}} \\
B
\end{array}\right] . \\
L=-B_{\text {input }, 1} B_{\text {input }, 2}^{-1}=-\frac{B}{\sqrt{2}} \times B=-\frac{1}{\sqrt{2}} . \\
A_{\text {sys }, 1}+L A_{\text {sys }, 3}=\frac{A-1}{2}+\left(-\frac{1}{\sqrt{2}}\right) \frac{A}{\sqrt{2}}=-\frac{1}{2}<0 .
\end{gathered}
$$

The next section describes the performance evaluation results that were obtained through experimental validations using an actual DC motor test platform.

\section{Performance Evaluation}

Figure 3 shows a model schematics for performance evaluations of the designed fault reconstruction algorithm.

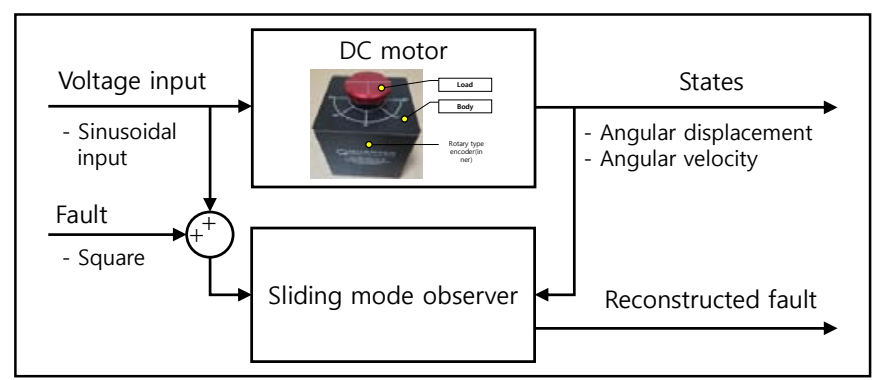

Figure 3. Model schematics for performance evaluations 
To obtain the angular velocity, the double integrator modelbased Kalman filter was applied using experimental data with processes and Gaussian noises. Two fault cases were set up to conduct performance evaluations by applying different squareshapes of voltage at 2-3 sec in simulation. A sinusoidal voltage input (amplitude: 1 volt, frequency: $0.2 \mathrm{~Hz}$ ) was applied to the DC motor test platform. Figs. 4-5 show the applied voltage input and fault signals in each case, respectively. The value of an equivalent injection term was calculated using a first-order transfer function with a time constant of $0.05 \mathrm{sec}$.

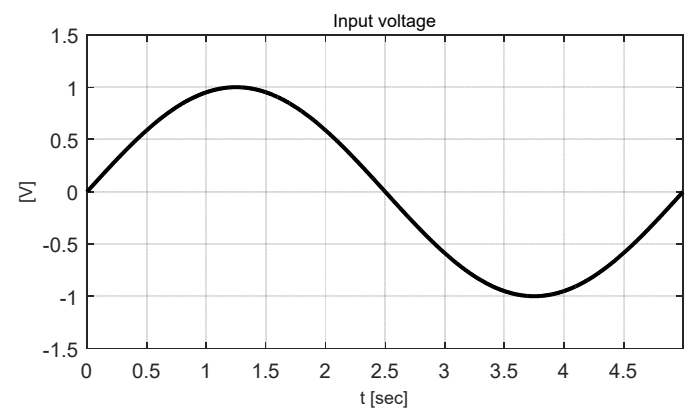

Figure 4. Sinusoidal voltage input used for performance evaluations

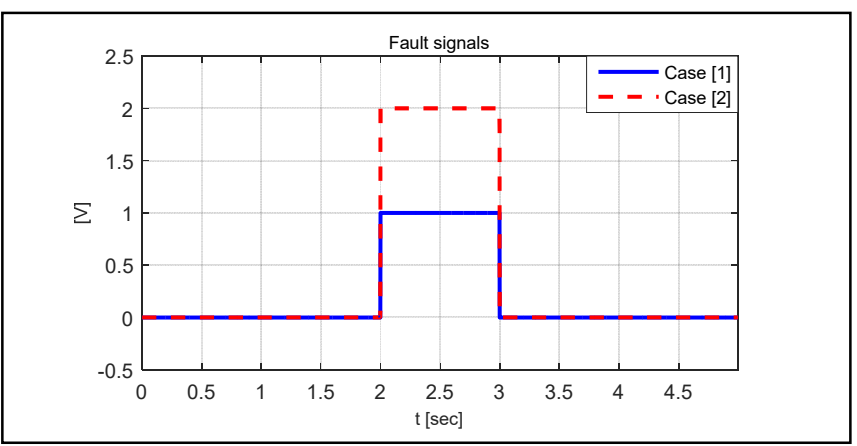

Figure 5. Fault signals of a voltage sensor applied in each case

Figs. 6-10 present evaluation results of the developed fault reconstruction algorithm.

Case 1: sinusoidal voltage input and square-shaped fault signal with an amplitude of 1 volt

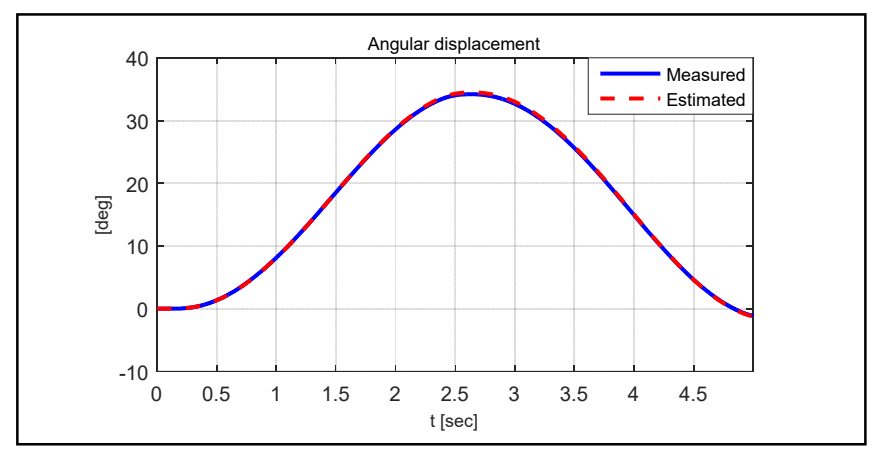

Figure 6. State estimation: angular displacement

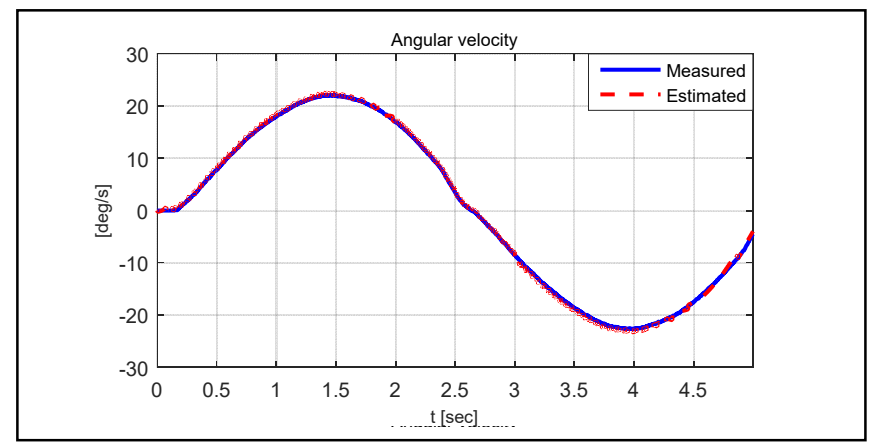

Figure 7. State estimation: angular velocity

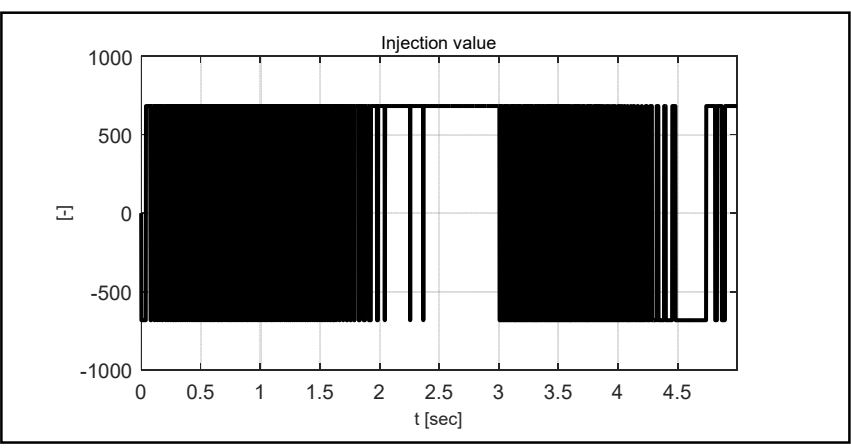

Figure 8. Discrete injection value

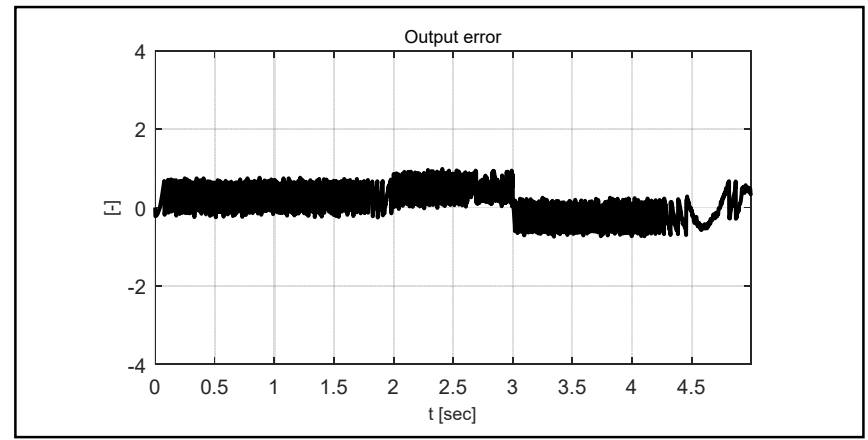

Figure 9. Output error

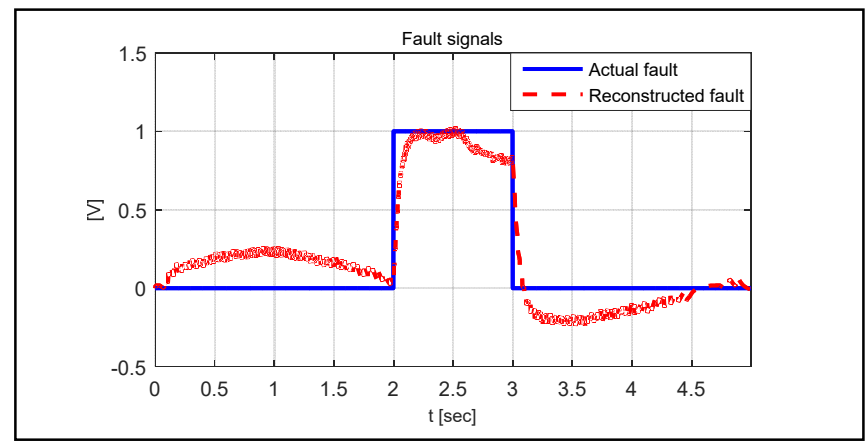

Figure 10. Fault signals: actual (blue) and reconstructed (dashed red) in case 1

Case 2: sinusoidal voltage input and square-shaped fault signal with an amplitude of 2 volt 


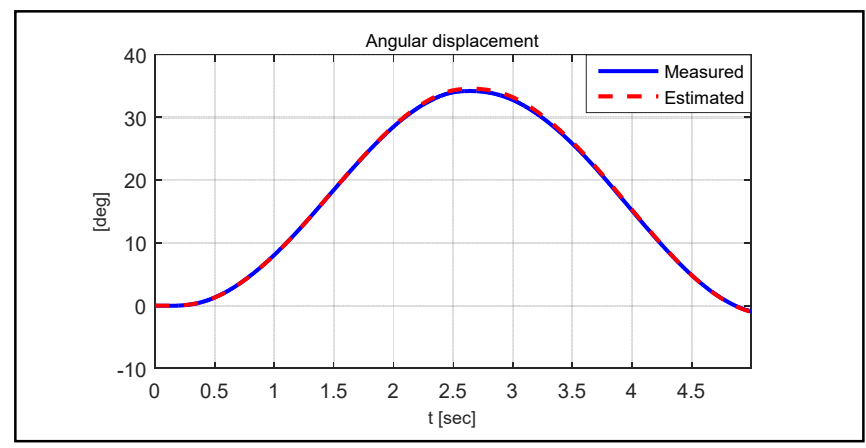

Figure 11. State estimation: angular displacement

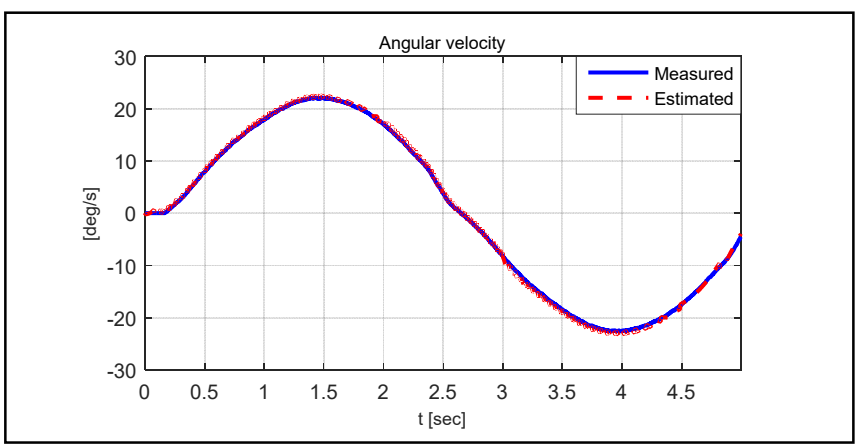

Figure 12. State estimation: angular velocity

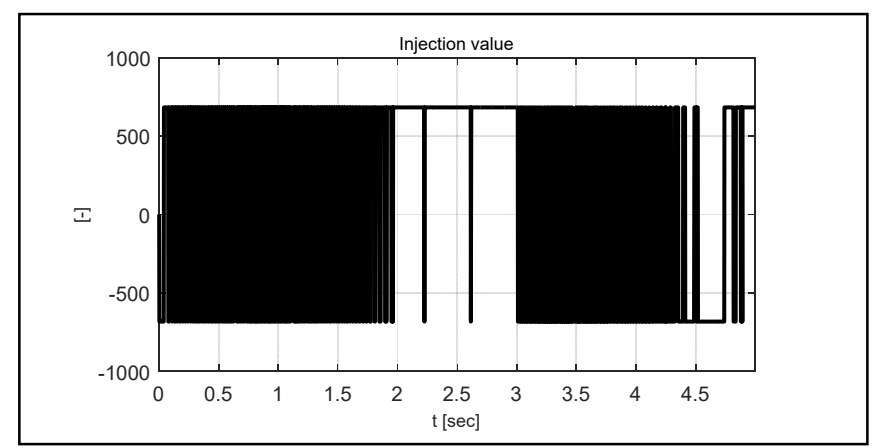

Figure 13. Discrete injection value

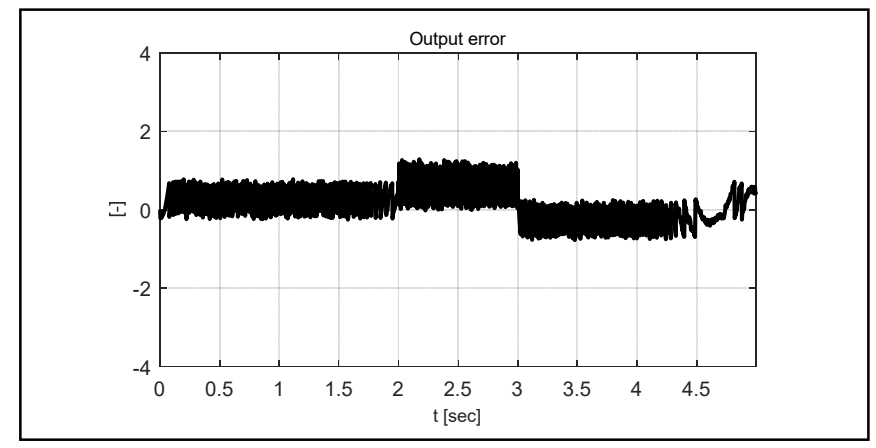

Figure 14. Output error

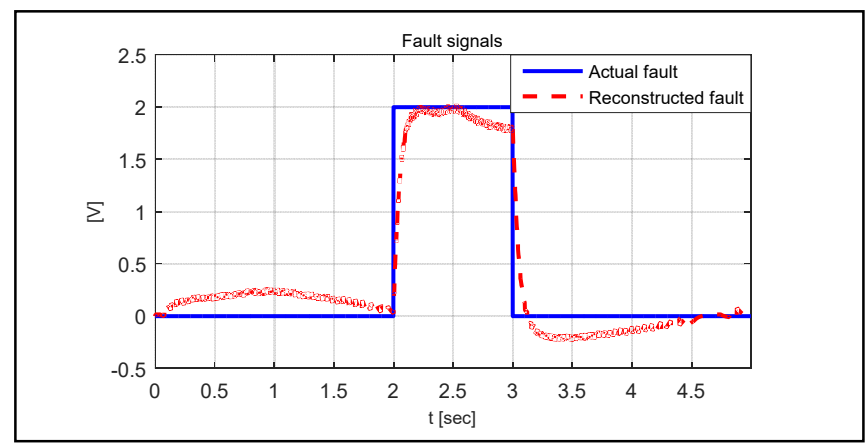

Figure 15. Fault signals: actual (blue) and reconstructed (dashed red) in case 2

As seen in Figs. 6-7 (case 1) and 11-12 (case 2), the designed sliding mode observer shows the reasonable performance of state estimation despite an occurrence of a fault signal during the simulations. In addition, Fig. 8 and 13 show that the switching frequency of an injection value for the designed sliding mode observer is very low while the fault is applied at 2-3 sec. In Fig. 9 (case 1) and 14 (case 2), it is observed that output errors vary between -1 and 1 and have a slight vertical shift at 2-3 sec. Finally, it is noted in Fig. 10 and 15 that the actual (original, blue line) signal can be reconstructed by the developed algorithm with a small range of error (i.e., \pm 0.25 between actual and reconstructed signals) except for the region of rising and falling edges. The concluding remarks are provided in the next section with future works.

\section{CONCLUSION}

This study proposes the sliding mode observer-based fault reconstruction algorithm for an input voltage sensor of the DC motor. In the proposed algorithm, a fault sensor signal was reconstructed using system parameters and an equivalent injection term for the sliding mode observer that was computed using a first-order transfer function. From test results using an actual DC motor platform in two fault cases, we can note that the developed algorithm has good capabilities for fault signal reconstruction and state estimation. However, since a small amount of error between the reconstructed and actual fault signals is still observed, reconstruction accuracy can be enhanced through improving the performance of the developed observer and filtering algorithms that will be considered as future work of this study. The design of a system identificationbased adaptive sliding mode observer can be another topic for a future extension since this method can contribute to the improvement of robustness and accuracy of fault reconstruction by dealing with load inertia variation. When considering the sound features of signal fault reconstruction, the proposed algorithm combined with the system identification techniques can be extensively used for fault detection and tolerant control of DC servo systems.

\section{ACKNOWLEDGMENT}

N/A

\section{REFERENCES}


[1] A. Adouni, A. Abid, and L. Sbita, "A DC motor fault detection, isolation and identification based on a new architecture artificial neural network," IEEE 2016 5th International Conference on Systems and Control (ICSC). Marrakesh, Morocco, pp. 294-299, July 2016.

[2] S. Lu, Q. He, T. Yuan, and F. Kong, "Online fault diagnosis of motor bearing via stochastic-resonance-based adaptive filter in an embedded system." IEEE Transactions on Systems, Man, and Cybernetics: Systems, vol. 47, no. 7, pp. 1111-1122, 2016.
[3] G. Singh, T. Kunmar, and V. Naikan, "Induction motor inter turn fault detection using infrared thermographic analysis." Infrared Physics \& Technology, vol. 77, pp. 277-282, 2016.

[4] S. Kommuri, J. Rath, and K. Veluvolu, "Sliding-mode-based observercontroller structure for fault-resilient control in DC Servomotors." IEEE Transactions on Industrial Electronics, vol. 65, no. 1, pp. 918-929, 2018.

[5] Y. Shtessel, C. Edwards, L. Fridman, and A. Levant, Sliding Mode Control and Observation, Springer Science \& Business Media, 2013. 